\title{
OCUPACIÓN HUMANA HOLOCÉNICA EN EL NORESTE DE LA MESOPOTAMIA: LA GRUTA TRES DE MAYO (GARUHAPÉ, MISIONES, ARGENTINA)
}

\author{
Antonia Rizzo, Aníbal Figini, Susana Salceda, Eduardo Tonni
}

\section{Introducción}

La Gruta Tres de Mayo se halla ubicada sobre la margen derecha del arroyo del mismo nombre, localidad de Garuhapé, Departamento Libertador General San Martín (Misiones), en un cauce pequeño que nace en las sierras centrales y desemboca en el Alto Paraná, presentando dos saltos producto de la erosión retrocedente en la meseta inmediata.

La superficie de la cueva es de aproximadamente $120 \mathrm{~m}^{2}$ y sus dimensiones lineales $40 \mathrm{~m}$ de frente, $30 \mathrm{~m}$ de profundidad y 7,50 $\mathrm{m}$ de altura en la boca de entrada. Su ocupación fue ininterrumpida entre los 0 y $\operatorname{los} 0,60 \mathrm{~m}$, primero sectorizada y posteriormente en su casi totalidad, con presencia de talleres líticos, cerámica, restos óseos animales, material conchífero, fogones y restos humanos.

De este sitio arqueológico se ha recuperado material lítico y cerámico, esqueletos humanos y numerosos restos faunísticos (vertebrados e invertebrados).

Recientemente se efectuó una datación radiocarbónica sobre restos faunísticos que permitió agregar un contexto cronológico preciso a este interesante sitio.

Para los estudiosos de las antiguas poblaciones humanas que habitaron el cono sur americano, una referencia cronológica, siempre abre un debate. Más aún si esa fecha marca una antigüedad, previamente sólo inferida como probable, y la cual aparece en escena siendo la protagonista por su profundidad temporal. Ni que decir si ello remueve antiguas asociaciones bioculturales.

Este es el caso de la Gruta Tres de Mayo. Un asentamiento humano en cueva, con una sorprendente variedad de instrumentos en piedra, hueso y concha, con cerámica lisa y restos esqueletarios humanos que, en el momento en que la entonces profesora Rizzo defendía su tesis doctoral, muy poco fueron tenidos en cuenta como diagnósticos de la presencia de un hombre temprano en territorio misionero.

En la presente contribución se resumen las principales características del sitio, discutiéndose su significado a la luz de la cronología absoluta.

\section{Hábitat y uso de los recursos}

La zona participa de las condiciones geológicas generales típicas de la provincia de Misiones, caracterizada por rocas volcánicas, restos de arenisca continental, tierras lateríticas, aluviones y sedimentos recientes. Edafológicamente los suelos son muy evolucionados manifestándose estratigráficamente de manera homogénea y en lo biogeográfico se corresponde con la flora y fauna propia de la selva climáxica. 
La materia prima utilizada para la confección de artefactos líticos fue el basalto y la arenisca silicificada. La frecuencia diferencial de elementos encontrados permite inferir que la industria de lascas con instrumental bien definido, fue preponderante. Es destacable la presencia de instrumental confeccionado en hueso (anzuelos, perforadores, puntas, agujas, adornos, entre otros). Algunas puntas óseas presentan decoración de líneas de puntos paralelas y su hallazgo permite inferir que pudieron ser utilizadas para la caza de pequeños animales. Del mismo modo, las agujas con ojo indicarían una incipiente industria textil.

Entre los restos de mamíferos hallados en el sitio se destacan algunas especies de mayor porte como el tapir (Tapirus terrestres) y la corzuela (Mazama sp.); entre los xenartros cingulados Cabassous sp. y Dasypus hybridus. Los hallazgos indican una selección de especies para la caza con una frecuencia relativa del $60 \%$ parar Tapirus terrestris y del $25 \%$ para Mazama $s p$. La presencia de gran cantidad de restos faunísticos con fractura intencional son indicativos de prácticas relacionadas con extracción de médula ósea. Los innumerables restos de peces, permite además inferir que el grupo complementó su dieta con pescado en gran escala.

Entre los invertebrados se menciona la presencia de moluscos acuáticos como Diplodon hylaeus (muy abundante), Diplodon parallelopippedon, Pomacea cananiculata y Aylacostoma guaraniticum y de gasterópodos terrestres como Strophocheilus oblongus (muy abundante). Esta presencia es indicadora de una actividad de recolección y evidente selección de especies para complementar la dieta, o para su uso en tareas artesanales (cuentas, pendientes, anzuelos), en especial y por su abundancia del Diplodon hylaeus.

Este conjunto faunístico no incluye señales de un posible cambio climático respecto de las condiciones actualmente imperantes en el área (véase Tonni, 2004)

Los fogones, ubicados en la parte central hacia el fondo de la gruta, están conformados por el agrupamiento de bloques de basalto que presentan rastros de su exposición al fuego.

Los caracteres composicionales y morfológicos de la alfarería, cuya presencia fragmentaria se detecta inmediatamente por debajo del nivel superficial, permite inferir su afinidad con la entidad agroalfarera regional Eldoradense.

\section{La datación radiocarbónica}

Se realizó una datación radiocarbónica (LP-1446) sobre colágeno de huesos de Tapirus terrestris, la cual brindó una fecha de $3550 \pm 60$ años radiocarbono A.P. Calibrada en años calendario (1 sigma) la fecha se ubica en el lapso 3721 a 3895 años calendario AP.

La fecha taxón ofrece un dato confiable acerca del momento de muerte del ejemplar fechado al tiempo que aporta datos sobre la totalidad del conjunto estratigráfico. La utilización de la proteína del hueso (colágeno) en lugar de otra fracción (por ej.: apatita) aumenta la confiabilidad del dato al reducir la interferencia de posibles contaminantes. Un hueso actual contiene aproximadamente un $20 \%$ en peso de colágeno; cuando el animal muere y sus huesos son incorporados al sedimento, se produce una gradual declinación 
del colágeno hasta llegar a desaparecer. Cuando se extrae colágeno de un hueso con un contenido menor al $1 \%$, la datación carece de confiabilidad dado que los efectos de los contaminantes son más significativos.

\section{Los restos óseos humanos}

Los cuatro esqueletos con estricta correspondencia individual, fueron rescatados en las cuadrículas H 7; I 9; J 7 y M 11 (Figura 1) y así serán nominados los individuos en este trabajo. Las características del ambiente en el cual fueron inhumados, además de la acción de agentes biológicos (actividad destructora del cohipí, insecto característico de la zona, sobre el hueso) y no biológicos (derrumbe de la pared rocosa) constituyeron factores negativos determinantes de un mal estado general de preservación de los materiales.

La posición mortuoria en la que fueron encontrados, salvo dos de los individuos en que sus huesos aparecen dispersos en un radio perfectamente definido (H 7 y J 7), es genupectoral, en uno de los casos sentado (I 9) y en el otro decúbito lateral izquierdo (M 11) orientados mirando al sur.

En la Tabla 1 se presenta el conjunto de elementos óseos craneanos y poscraneanos correspondientes a cada uno de los individuos.

El análisis osteológico en concordancia con las técnicas convenidas internacionalmente, permitió realizar la asignación de edad y sexo a cada individuo con la precisión estimativa que la naturaleza del material preservado determinó. Estamos en presencia de tres individuos adultos, dos de ellos masculinos (H 7 y J 7 ) y uno femenino (I 9). El cuarto individuo es maduro y de sexo masculino (M 11)

En la Tabla 2 se muestra los resultados del relevamiento métrico realizado sobre los esqueletos que por encontrase en mejor estado de conservación así lo permitieron.

\section{Discusión y conclusiones}

Es válido imaginar el escenario en esos tiempos, sobre todo teniendo en cuenta que los actuales límites políticos entre los países sudamericanos no constituían entonces las reales barreras que separaban a esas antiguas poblaciones de cazadores recolectores. En la síntesis sobre prehistoria de Brasil de Funari y Noeli (2001), en particular cuando hacen referencia a la creciente diversidad de los antiguos habitantes, se permiten sugerir siguiendo a Walter Neves, la existencia de tres etapas de ocupación humana en ese territorio. La primera muy poco conocida, que corresponde al llamado sustrato paleoindio (hasta los 12.000 años AP); la segunda estaría marcada por una colonización mongoloide desde el noroeste de América del Sur, que se esparció rápidamente por el litoral sudamericano y con mayor lentitud hacia el interior del territorio (hasta los $9.000 \mathrm{AP}$ ), y la tercera etapa que tiene su origen en pueblos mongoloides asentados en Amazonia portadores de agricultura, cerámica y nuevos patrones demográficos y culturales de economía y organización social. En esta extensa etapa, especialmente entre los 4.000 y 2.000 años AP (según los arqueólogos Lathrap y Brochado) una gran explosión demográfica en Amazonia fue la causante de la 
expansión geográfica de estos pueblos ceramistas y agricultores que sustituyeron a las poblaciones cazadoras-recolectoras según la postura de Neves compartida por los autores.

Hacia el sur, para la Región Pampeana, la cronología y características de las ocupaciones de los pueblos ceramistas y sus correlaciones extrarregionales han sido minuciosamente analizadas por Politis et al. (2001) quienes marcan tres momentos de incorporación de esta innovación tecnológica, secuencialmente correlativos, que involucran distintos espacios geográficos y por lo tanto permiten seguir una "ruta" de dispersión a quienes comparten el criterio del carácter foráneo de la emergencia de esta tecnología: sudeste de la Región Pampeana, norte y noreste de Uruguay, entre los 3000 y 2500 años AP; noreste de la Región Pampeana y litoral del Río de La Plata, entre los 1700 y 1500 años AP y norte de Patagonia entre los 1500 y 1200 años AP. Los autores consideran la multifactorialidad en el surgimiento de la complejidad social y del proceso de intensificación planteando para la región, factores que abarcan mucho más que aquellos ligados exclusivamente a la subsistencia y a la economía.

La Mesopotamia misionera ofrece también antiguos registros de industria lítica (Huamitá, Ivaí, Uruguay II) con profundidades temporales superiores a los $4000 \mathrm{AC}$ (Rodríguez y Ceruti, 1999). Salto Grande, ya en territorio entrerriano, constituye una entidad en la cual la gradual transición de Ivaí a la presencia de grupos humanos con manejo de la tecnología cerámica y con dataciones que la remontan a 500 años AC se transforma en un referente a tener en cuenta en esta oportunidad. Grupos humanos con un manejo acabado de la tecnología lítica que dejan pruebas de la presencia de los primeros vestigios de alfarería.

Es en este marco espacio temporal, en el que la Gruta Tres de Mayo adquiere significación, no sólo porque provee el fechado más antiguo para la provincia de Misiones, sino también porque muestra una secuencia precerámico-cerámico en sus dos niveles de ocupación continua, un uso creciente del espacio por un aumento demográfico, y por lo tanto una complejización social, y la presencia de una tecnología sofisticada en la elaboración de artefactos.

Aquí, en clara asociación con el precerámico, 2000 años antes de Cristo, se asentó un grupo humano cuyas características biológicas adquieren hoy una significación particular, más allá de las exhaustivas comparaciones morfológicas que allá por la década del 60' permitieron a Lilia Chaves de Azcona incluirlos en el grupo de los Amazónidos de Imbelloni (Brasílidos de von Eicksted) y considerarlos ancestros de los guayaqui etnográficos por sus características bioantropológicas.

Las nuevas evidencias refuerzan la hipótesis que desde hace 4000 años aproximadamente, vivieron en la Selva misionera pueblos cazadores, recolectores, pescadores, con manejo de técnicas cerámicas. Estos pueblos estaban integrados por individuos de mediana a pequeña talla, débilmente dimórficos, con cabezas redondeadas y altas, frente media con crestas divergentes, de órbitas altas, nariz baja y muy ancha y paladar corto y ancho, portadores de una industria en piedra, hueso, concha y alfarería fina y lisa que se corresponden con aquellas poblaciones referidas en la bibliografía como neoamerindias. Estas poblaciones ocuparon preferentemente las regiones forestales, en 
la proximidad de los grandes ríos y sus afluentes, con una amplia dispersión geográfica con peculiaridades regionales, estilísticas y tecnológicas. Sus vastas migraciones en el continente sudamericano han motivado la presencia de variaciones locales, según las peculiaridades ecológicas de cada ambiente y los mestizajes con poblaciones anteriores (Salceda y Méndez, 1999).

El sitio Tres de Mayo fue un lugar de ocupación humana desde tiempos los comienzos del Holoceno tardío. Su estratégica ubicación, la potencialidad sustentable del medio ambiente natural, la cercanía a los cursos de agua para el desplazamiento, lo constituyeron en un lugar por demás apto para la subsistencia de los grupos humanos que inicialmente fueron aceramistas y más tarde alfareros.

\section{Planta - Gruta 3 de Mayo}

\section{Distribución espacial de restos humanos}

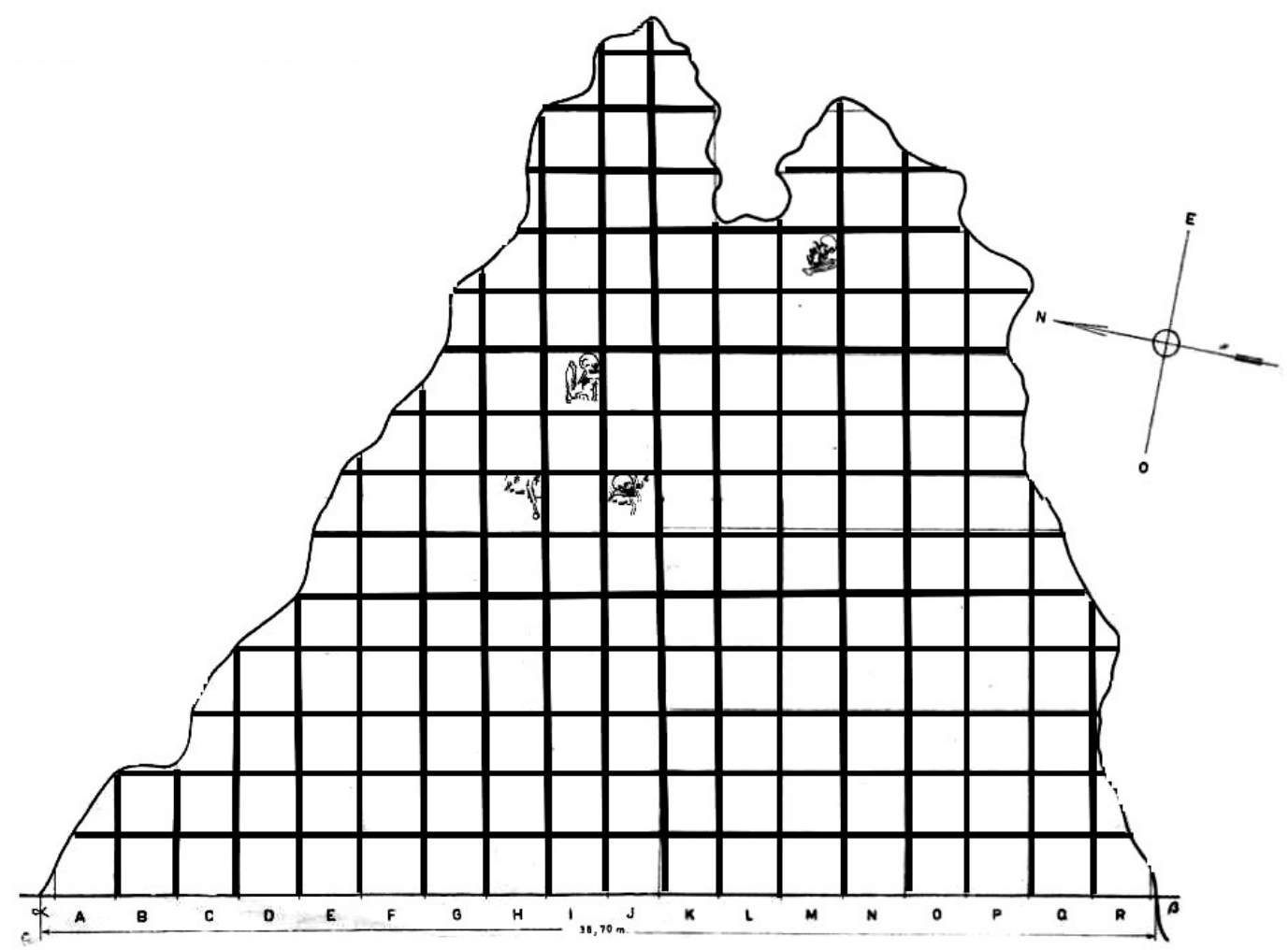


Tabla 1

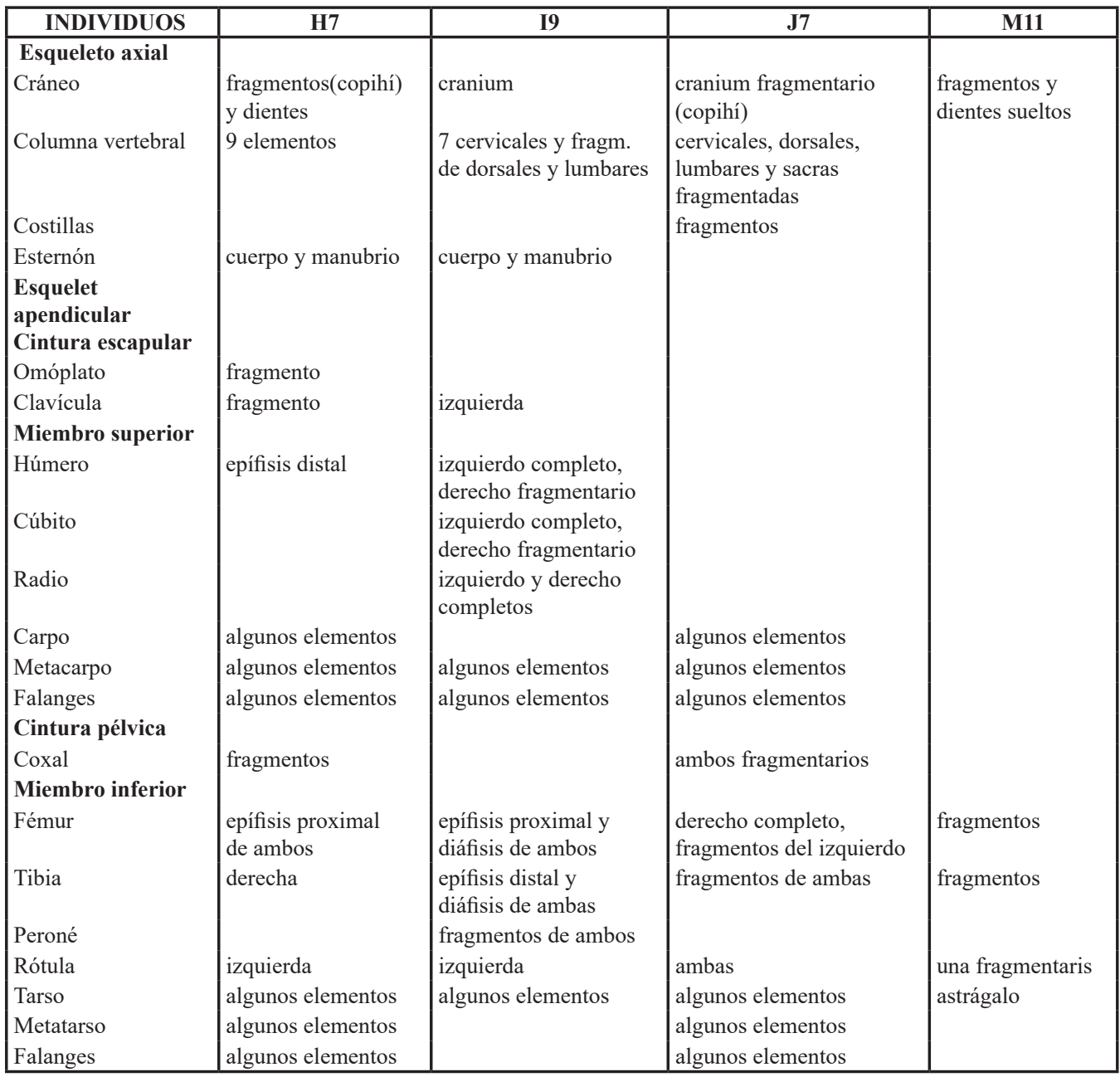

Tabla 2

\begin{tabular}{|c|c|c|c|c|}
\hline INDIVIDUOS & \multicolumn{2}{|c|}{ I9 } & \multicolumn{2}{|c|}{$\mathbf{J} 7$} \\
\hline INDICES CRANEANOS & Valor numérico & Caracterización & Valor numérico & Caracterización \\
\hline Craneano horizontal & 78.92 & mesocráneo & 78.57 & mesocráneo \\
\hline Vértico longitudinal (auricular) & 63.25 & hipsicráneo & 68.45 & hipsicráneo \\
\hline Vértico transversal (auricular) & 80.15 & metriocráneo & 87.12 & acrocráneo \\
\hline Frontal transversal & 80.91 & intermedias & 81.31 & divergentes \\
\hline Fronto parietal & 67.94 & metriometope & 65.91 & stenometope \\
\hline Nasal & 60.9 & hiperplatirrino & & \\
\hline Orbitario & 91.6 & hipsiconco & & \\
\hline Palatino & 91.6 & braquiestafilino & & \\
\hline INDICES POST-CRANEANOS (Fémur) & & & & \\
\hline Platimérioco & 97.29 & & 75.0 & hiperplatimería \\
\hline Pilástrico & & & 108.7 & pilastra débil \\
\hline De robustez & & & 18.3 & poco robusto \\
\hline ESTATURA (en cm) según Pearson & & & 161.77 & media \\
\hline
\end{tabular}




\title{
Bibliografía
}

Funari P.P. e Noeli S.F., 2001. Pré-História do Brasil. Ed. Contexto. São Paulo. Brasil.

Politis G.G., Martínez G.A. y Bonomo M., 2001. "Alfarería temprana en sitios de cazadores-recolectores de la Región Pampeana (Argentina)". Latin American Antiquity, 12(2): 167-181.

Rodríguez J.A. y Ceruti C.M., 1999. "Las Tierras Bajas del Nordeste y Litoral Mesopotámico". En: Nueva Historia de La Nación Argentina. Academia Nacional de la Historia. Ed. Planeta. Cap. 4: 109-131.

Salceda S.A. y Méndez M.G., 1999. “La biodiversidad amerindia”. En: Nueva Historia de La Nación Argentina. Academia Nacional de la Historia. Ed. Planeta. Cap. 2: 65-82.

Tonni, E.P., 2004. "Faunas y clima en el Cuaternario de la Mesopotamia argentina". En F. Aceñolaza (ed.): Temas de la biodiversidad del litoral fluvial argentino. INSUGEO, Miscelanea 12:31-38.

\section{Resumen}

La actualización y revalorización de la información derivada del análisis de los materiales bioculturales procedentes de las excavaciones realizadas en 1965 en la Gruta 3 de Mayo, adquieren hoy especial significación al contar con la cronología radiocarbónica del sitio (LP -1446: $3550 \pm$ $60 \mathrm{AP}$ ). Esto genera un marco contextual para la interpretación de situaciones de contacto, cambio de la organización social y modos de vida, entre otros aspectos, que en su conjunto, y ante la ausencia regional de yacimientos estratigráficos de amplio lapso de ocupación, representa un valioso reservorio cultural secuencial.

$<$ Poblamiento temprano $><$ Noreste Mesopotamia $><$ Cazadores-recolectores $>$

\begin{abstract}
In this paper is carried out an upgrade of the information coming from the biocultural materials found in 1965 at the Gruta 3 de Mayo (Garuhapé, Misiones). The radiocarbonic chronology of the site (LP -1446: $3550 \pm 60$ radiocarbon years BP) allows to generate a background for the interpretation of contact situations, changes in the social organization, and ways of life.
\end{abstract}

$<$ Early peopling $><$ Northeastern Mesopotamia $><$ Hunter-gatherers $>$ 